\title{
Overview of Licensing Platforms based on Distributed Ledger Technology
}

\author{
Alexander Schoenhals ${ }^{1}$, Thomas Hepp ${ }^{1}$, Stephan Leible ${ }^{2}$, Philip Ehret ${ }^{1}$, and Bela Gipp ${ }^{1}$ \\ ${ }^{1}$ Department of Computer and Information Science, University of Konstanz * \\ ${ }^{2}$ Department of Business and Industrial Engineering, Offenburg University of Applied Sciences ${ }^{\dagger}$
}

\begin{abstract}
The licensing of creative work is of broad and current interest. The European Commission proposes that when uploading a licensed digital work, the uploader should be checked by the system that one has the necessary rights. Technically this law is difficult to implement, as images with different intentions are shared, and even small changes like watermarks make it difficult to reveal similarities. The characteristics of distributed ledger technology could provide excellent support for the licensing and management of the rights of use. In this work, non-technical and technical criteria are defined to achieve an overview of the state-of-the-art solutions in the field of blockchain-based licensing platforms. Based on the criteria, different licensing platforms are reviewed, and the results are presented in a comparison matrix.
\end{abstract}

\section{Introduction}

Developing new ideas and novel concepts is one of the fundamental steps to success in both science and industry. Therefore, the importance of tracking these inputs to reward the author in the case of commercial use or to share in the profit is raising.

Due to increasing prosperity, but also due to globalization and digital networking, the potential for innovation has increased enormously worldwide in recent decades. While a constant 1 million patent applications were disclosed annually between 1985 and 2000, a continuous growth rate of $10 \%$ has been recorded since the turn of the millennium - in 2016 over 3.1 million patents were disclose ${ }^{1}$

Proving the authorship of already patented technologies and concepts is well implemented through the rigorous patent process. This traditional process is centralized which means there is one authority that

* \{firstname.lastname $\}$ @uni-konstanz.de

† ste.leible@gmail.com

1 https://www3.wipo.int/ipstats/ decides on the acceptance or rejection of a patent application. In this case, the authority can be corrupt or subjective, so a fair decision isn't guaranteed. The situation is different with digital content. To license digital work, the Creative Commons was founded in 2002. By 2016, over 1200 million works had been licensed with the help of Creative Commons standard. However, the problem with licensing remains in its traceability. Most authors cannot prevent the re-use of their works because it is often not possible to prove their authorship. The problem: Digital documents can be copied and duplicated easily [1]. And even if a label or watermark is used, e.g., a Creative Commons marking, it can be removed.

However, licensing and consistent tracking of digital assets is a definite requirement resulting from several surveys and approaches [2, 3, 4] on systems related to Open Innovation [5]. It is indispensable that a clear usage regulation of submitted digital assets must be integrated into the distributed computer-based system to open the innovation processes consequently [6].

Copyright infringement is a huge problem and leads to significant annual damages. Digital rights management tries to solve these piracy problems and also deals with origin issues of particular digital assets. The main focus is on ensuring that content creators get paid for their work. Due to the intransparent traceability, it is difficult for the distribution platform operators to ensure this financial obligation towards the originator.

A decentralized approach has the potential to address these challenges. Not only for the originator, but also for leading distribution platform operators such as Apple, Google, Netflix, and Amazon.

\subsection{What is a Blockchain?}

The blockchain is a distributed data structure that maintains a continually growing linked list of publicly accessible records. The records, stored on-chain, are tamper-proof and cryptographically secured from revision [7]. Further, it is evident that the traits 
of Open Innovation, like distribution and publicly open access, go hand in hand with the attributes of Distributed Ledger Technologies (DLT), e.g., the blockchain, directed acyclic graphs [8]. Since the Bitcoin white paper in the year, 2008 [9], a lot of work has been done in the field of decentralized storage and computation [10]. Furthermore, it has already been described that DLTs can be used not only for a transaction of cryptocurrencies but also for registering and confirming of contracts and properties, e.g., by using trusted timestamping [11, 12]. Mainly, this is due to the following key characteristics of DLT:

Decentralization. Completely decentralized - one doesn't have to trust the system's individual but the system on the whole.

Immutability. If data is recorded once on the distributed ledger, it cannot be reversed. This attribute makes a submitted asset on the ledger traceable.

Accessibility. Blockchains allows anybody who is a part of the network to read any data recorded by anybody.

The popularity of decentralized systems and especially blockchain causes a broad range of literature, although the topic, in general, is a strongly evolving field. Even if very versatile concepts are described, most implemented solutions just focusing on notarization and registration of digital documents [11, 13] and less on distribution and traceability of licensed assets stored or referenced using the blockchain technology (BT).

Nevertheless, more and more blockchain applications are being created, which not only confirm the proof-of-existence of digital assets but also regulate the use of data through licenses controlled by defined smart contracts on more complex platforms, i.e., user and identity management, access control [14], audit of smart contracts ${ }^{2}$, storage. Reason enough to investigate what the challenges are and what added value such newly emerging systems offer for the opening of innovation processes.

\subsection{Licensing}

Intangible goods such as inventions, creative works or even brands have constantly gained in economic importance since the age of industrialization. Accordingly, issues of legitimacy and scope of legal protection of these intangible assets have been and will be discussed as intellectual property (IP). Efforts to effectively protect IP rights have intensified in recent years as their economic value was recognized.

2 https://solidified.io/
A license agreement enables the licensee to establish a right of use legally. Not the licensing is the big challenge, but the proof of a right of use - proof that someone is using IP without rights. The right to use digital documents once published on the Internet is difficult and, above all, costly to verify. The characteristics of the Distributed Ledger Technology could greatly facilitate traceability for licensors and licensees. Not only startups but also governments are facing this possibility [15, 16].

\subsection{Outline}

The objective of this paper is to provide a state-of-the-art analysis of solutions which are focusing on licensing and copyright protection on the basis of DLT/BT. What are the specific problems and characteristics of different applications that provide blockchain-based licensing of digital assets?

We identify and describe the differences and similarities, strengths and weaknesses and show them in a comparison matrix based on defined criteria. On the one hand, we want to monitor future directions, and on the other hand, we want to reveal the chances and limitations of state of the art.

The remainder of this paper is organized as follows. Section 2 covers the previous approaches on how blockchain-based applications were classified. The description of the selection process, as well as comparison criteria, is presented in Section 3 Selected applications are briefly introduced in Section 4 A discussion based on the results of the comparison matrix is presented in Section 5 . Finally, Section 6 summarizes the paper and suggests future directions.

\section{Background}

In the following section, we will discuss related work dealing with the classification of different blockchain applications. The classification of applications is necessary to narrow down the scope due to the broad mass of various applications.

As de la Rosa et al. has already mentioned, the nature of Open Innovation corresponds to the nature of BT [2]. According to de la Rosa et al., one finding was that industry is trying to open up its innovation processes.

However, bringing together these different interests to live the Open Innovation paradigm are big challenges. It's about uniting different players who have to work together in a trustful and efficient manner for the right reasons. In their survey, they describe characteristics that must be integrated into a platform to address these challenges. Relevant features also for our examination 
are, e.g., record keeping, citation traceability, licensing but also rewarding mechanisms.

De la Rosa et al. present various services and compare them based on defined criteria. Their research of the platforms focuses on enabling of Open Innovation for small and medium-sized enterprises (SME). They note that BT will have an enormous impact on Open Innovation but is still in an 'embryonic' phase. This leads to the consequence that one must continuously observe the development since almost monthly new approaches/platforms are launched/announced.

In summary, their work is essential and useful, but the given overview is high-level and does not go into the technical details, beyond that, important features like identity management or licensing types are not shown in detail.

However, the authors give a very good and detailed look into the future. According to de la Rosa et al., BT is capable of becoming a mainstream technology for Open Innovation ecosystems.

Elsden et al. [3] and Kane [17] published further classifications of different blockchain applications. While Kane provides a general overview of the applications and sorts them into the classes defined by Swan [18], Elsden et al. create its typology.

Swan separates blockchain applications into three different versions: Blockchain 1.0, 2.0, and 3.0. Blockchain applications are divided into currencies, contracts, and applications beyond finance. Thus, Blockchain 3.0 applications are holistic and cover complex activities.

At the beginning of 2017, Kane classified three-quarters of about 200 examined applications as Blockchain 2.0 applications, e.g., financial services, basic crowdfunding platforms, and IP registries.

In our work, we deal with Blockchain 3.0 applications, with a more pervasive approach to identity management, distribution, and license-based access control.

Elsden et al. investigate in their work the influence of existing but also emerging blockchain applications on the field of human-computer interaction (HCI). Therefore, a typology is being developed to answer topics of interest to their field, e.g., usability, trust, and user-friendliness. Nevertheless, the structure is also useful for us. They divide the typology into seven classes: Underlying infrastructure, currency, financial services, proof-as-a-service, property and ownership, identity management and governance (see Table 1). If one would overlay the classification of Elsden et al. and Swan, the intersection between Blockchain 2.0 and Blockchain 3.0 applications would be property and ownership. Applications that belong to that

\begin{tabular}{|c|l|}
\hline Application & \multicolumn{1}{|c|}{ Description } \\
\hline $\begin{array}{c}\text { Underlying } \\
\text { Infrastructure }\end{array}$ & $\begin{array}{l}\text { Underlying protocols, } \\
\text { decentralized application } \\
\text { ecosystems, IoT architecture. }\end{array}$ \\
\hline Currency & $\begin{array}{l}\text { Payment services, internal } \\
\text { currencies and utility tokens. }\end{array}$ \\
\hline $\begin{array}{c}\text { Financial } \\
\text { Services }\end{array}$ & $\begin{array}{l}\text { Asset management, } \\
\text { investment trading, and } \\
\text { crowdfunding. }\end{array}$ \\
\hline $\begin{array}{c}\text { Proof-as-a- } \\
\text { service }\end{array}$ & $\begin{array}{l}\text { Notaries, registers and } \\
\text { attestation, supply-chain } \\
\text { management. }\end{array}$ \\
\hline $\begin{array}{c}\text { Property and } \\
\text { Ownership }\end{array}$ & $\begin{array}{l}\text { Digital rights management, } \\
\text { copyright and ticketing } \\
\text { services. }\end{array}$ \\
\hline $\begin{array}{c}\text { Identity } \\
\text { Management }\end{array}$ & $\begin{array}{l}\text { Self-sovereign digital identity, } \\
\text { and authentication. }\end{array}$ \\
\hline Governance & $\begin{array}{l}\text { Voting services, distributed } \\
\text { autonomous organisations } \\
\text { (DAO's). }\end{array}$ \\
\hline
\end{tabular}

Table 1. Typology of seven classes of blockchain applications defined by Elsden et al. [3]

class represent complex ownership rights and regulate re-use of registered assets. The main difference to proof-as-a-service platforms are the additional functions of automated management of licensed documents with the help of, e.g., identity management.

We deduce that licensing and distribution platforms belong to the category property and ownership. In this study, only the approaches that can be classified in this category are examined.

\section{Method}

Since DLT is an emerging technology and in particular because of the media hype, more and more entrepreneurs are jumping on this hype and develop and offer distributed ledger approaches. It is a challenge to get a complete overview of existing solutions, especially since fraudulent companies [19] are also among the platforms. Elsden et al. have disclosed a lis ${ }^{3}$ of 204 solutions, but by far not all relevant platforms are included. Especially as new solutions appear on the market every month.

Our focus is on licensing and distribution platforms for creative work and intellectual property. Therefore,

3 https://doi.org/10.6084/m9.figshare. 5765502 .v1 (accessedon05/26/2018) 
we only consider approaches that can be assigned to the mentioned classes property \& ownership [3] and Blockchain 3.0 [20]. Furthermore, in this subgroup, we concentrate on the solutions that enable the licensing of input data and offer a distribution platform to control compliance with the licensed content.

As already mentioned, screening different applications is difficult. It must be distinguished in which development stage the app already is. This stage often becomes clear only through in-depth analyses. Sometimes it turns out that the solutions are merely concepts without any detailed implementation plans. To be able to make specific statements about the relevance of a solution, soft criteria, as well as technical criteria, were defined.

The non-technical criteria are primarily aimed at the user's perspective and should reflect what type of interfaces are provided and which functions can be used. These are, e.g., the access methods or the offered license types. Concerning the technical criteria, we would like to shed light on the foundation of the particular solution and understand the propagated IP protection methods. It is crucial to distinguish on which distributed ledger type the service is based or how the identity management is accomplished. We think that the criteria used help us to get a more detailed view of the individual solutions.

The defined criteria served as a guideline for reviewing the individual solutions. Even during the telephone calls with those responsible for the applications, these individual points gave us a structure for the interviews.

All criteria will be explained briefly below. First, the reasons for the different points are described and second, the various options available for each criterion are listed.

\section{Non-Technical}

Document Type. This attribute describes the kind of data which is intended to be protected and distributed. The different kinds of data can be for, e.g., images, videos, written ideas, and even IP patent files.

Business Type. That characteristic defines whether the customers are businesses or consumers. Business-to-Business (B2B) means that a business makes a transaction with another business. For example, when one company instructs the other company to build a new production hall for them, this case is fulfilled. One other business model is Business-to-Consumer (B2C) in which a company is selling their products to the consumer. This case happens in every supermarket or local store. If users use a licensing service then there is a B2C type. If we have a community then it is $\mathrm{C} 2 \mathrm{C}$ (Consumer-to-Consumer) and if we have business consortia then the type is marked as B2B.

Users range/Number of Users: An important category to measure the impact of the application. We suppose that the impact of an application is small when there are just a few dozen users active. If we find thousands of users the platform is not only well-known, it is also popular and provides more likely some innovating and useful features. To find out the number of users we orientate ourselves on the figures given by the companies, the amount of already licensed files or alternatively on the followers of social media accounts. Classification is given in low, mid and high - considered among each other in the respective business types.

Access methods. This criterion marks which access method a certain platform offers and whether they are freely accessible. An API allows to access contents and interact with the platform programmatically and hence facilitates various use cases, e.g., embedding the platform in a custom application. A web, mobile or desktop application rather addresses non-technical users and allows to explore the platform's contents with a graphical user interface.

License standards. All considered platforms have integrated licensing in some form into their concept. The platforms offer industry-specific standards for copyright and general usage rights. If the applications use standard licenses, we explicitly name them here, e.g., Creative Commons (CC), GNU General Public License (GPL), and governmental institutions (i.e., U.S. Copyright Office). If no standard is explicitly mentioned on the platform, we mark it as "own".

Audit smart contracts: In this category, we examine if there are certificates of the applications from smart contracts auditing providers. With these audits, the smart contracts of an application getting checked for bugs, security issues, and other possible mistakes, that application programmers have made in the programming of their smart contracts. The auditing company would issue a certificate for the checked-application regarding positive publicity if the customer solved all found mistakes.

\section{Technical}

Blockchain scope. The choice of the scope of a blockchain plays an essential role for the trust of the data [21]. On the one hand, public blockchains are considered trustful as Bitcoin and Ethereum have successfully demonstrated [22, 23]. On the other 
hand, private blockchains can be used flexibly, with higher performance and lower costs [24]. However, the operators of a blockchain can easily manipulate the data. But, if the blockchain allows the subsequent modification of data records, it endangers the trust of the underlying license. Subsequent manipulation destroys the advantage of decentralization. As a compromise consortium blockchains were presented, which should combine the strengths of both approaches [21]. Behind the network is a community with similar interests, but also, for example, different companies competing with each other.

Data structure. Blockchain, DAG, GHOST, Segregated Witness Apart from linked blocks, multi-data structures, like graphs, were evaluated and developed [21]. For example, IOTA uses directed acyclic graphs (DAG) [8] to map transactions in the network [25]. The different data structures try to improve the scalability of public blockchains, such as Blockclique [26].

Item Storage. Xu et al. also propose the classification of the item storage [21]. In order to archive documents in a tamper-proof manner, the data must be backed up in the blockchain. For this purpose, data can be embedded in a transaction or Smart Contracts can be used [24]. However, on-chain storage is de facto not applicable due to the high costs and the limited block size. Data can also be managed off-chain, which is significantly cheaper and more efficient [24, 21, 27]. Therefore various protocols were developed to profit from these advantages, such as the Lightning Network [28].

Consensus Protocol. The protocol controls how network participants are making decisions by consensus. That means not a single individual decides which way will be gone, it is a decision of everyone who is taking place in the network. The most common consensus protocols are "Proof-of-Work" (PoW) [9] and "Proof-of-Stake" (PoS) [29]. The former was invented by the pseudonym Satoshi Nakamoto within Bitcoins whitepaper [9]. In a PoW system miners generating new blocks that are on the one side performance costly and time-consuming, but on the other side, it is easy to verify by the network to generate consensus. PoS works with validators, who are chosen in a deterministic way (depends on their stake of coins in the network) to create new blocks. As consensus mechanisms exist a multitude of other systems such as "Practical Byzantine Fault Tolerance" (PBFT) [30], "Delegated Proof-of-Stake" (DPOS) [30], Sharding [31] and "Proof-of-Elapsed Time" (PoET) [31.

Reference Implementation. The underlying structure of the applications can be, for example, the Bitcoin or Ethereum blockchain. All characteristics of the used basis are coming with the corresponding blockchain, like transaction speed, consensus protocol, programming language, tokens, and APIs.

Identity Management. An essential factor in a solid binding of a licensed file to an originator is the type of identity management. There are conventional approaches that store user data in a centralized database and keep the authorization management centralized as well. Also, there are decentralized solutions in which the user data is stored distributed and verification of whether a user has access to a certain file or not is determined by consensus ${ }^{4}$ In the past, also hybrid solutions of identity management were presented, in which personal user data is stored in a database, but authorization is managed distributed.

Incentives. Incentives shall motivate all network participants to follow the underlying protocol. Most of the time incentives realized by the issue of tokens. If a users behavior is in harmony with the protocol, he gets rewarded, e.g., by tokens [24]. These have a monetary value, so there is amongst other things an intrinsic motivation to take part in the network. Incentive systems differ from application to application. We tag whether users of the platform receive incentives or not.

Peer-reviewed. This category includes independent reviews of individuals who are competent in the underlying field. It is a quality-control system in which in most cases other scientists scrutinize and criticize discoveries, implications, and ideas before they become widely published. If a concept or platform in our survey has been peer-reviewed, we will mark it with "yes", if not, it is marked with a "no".

\section{Overview of Applications}

This section is intended to summarize the applications considered. We describe both, the underlying technology and the functional scope of the platform. We have limited ourselves to the platforms that offer to license and manage the authorized data. The information was partly extracted from semi-structured interviews, but also from provided whitepapers.

\section{1. ascribe}

The Platform ascribe.io is designed for artists, creators, marketplaces, and developers. The platform provides different features for each of the stated

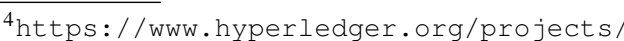
hyperledger-indy 
target groups, such as content protection by creating a digital fingerprint (proof-of-existence) with BT over the Bitcoin protocol and an image similarity search to track the prohibited usage of copyright data on the internet for artists, creators via web app, and external marketplaces. A commercial license contract does not protect content, i.e., a company that watches the usage of copyright content and gets a cut of the profit when the artist sells his work [32]. Ascribe also delivers a REST API for developers to implement the previously mentioned functionality into other apps or websites. Communication with the Bitcoin blockchain comes with their own protocol named SPOOL which registers a piece of work or transfers copyright rights between individuals and creates various outputs like hash information containing the work and metadata like the artist name, the title, and the year [32]. The service works for free. Also, there is no currency like ascribe tokens.

\subsection{Bernstein}

Bernstein focuses on securing industrial and technological property assets using BT. It is targeted towards intellectual property owners that aim to make defensive IP disclosures and allows to prove existence, integrity, and ownership of certain IP [33]. Users can upload IP assets via a web application; programmatical access is currently not possible. An asset's hash is stored in the Bitcoin blockchain, and the encrypted original file on a cloud server, the encryption's private key remains secret with the user. Bernstein explicitly does not aim to license, distribute and monetize assets, it focuses on ownership claims and therefore collaborates with a law firm who parallelly registers assets notarially [2]. Further technical details remain undisclosed.

\subsection{Binded}

Binded is based in the USA and is intended exclusively for the copyright protection of images. Users can either upload their pictures directly to the platform or connect social media platforms, e.g., Instagram. A digital fingerprint of the file is created and stored on the Bitcoin blockchain. The upload checks whether it is an image file. The image file is stored centrally in a database.

As soon as the user has uploaded an image via the web application, an algorithm is initialized in the background, which detects possible copyright infringements on the Internet. An exact description of that search algorithm is not known. We have uploaded a popular and licensed (CC) image for testing purposes, but no license violation was found. That proactive monitoring feature still has beta status.

Every uploaded file is kept in the so-called vault and continuously monitored. As soon as a violation of the rights is detected, there is a real-time notification that pops up within the web application.

In addition to the licensing by Binded, a copyright application can be started at the U.S. copyright office. It is offered for a fee of 35 USD. Otherwise, the use of service is for content creators free.

\subsection{Creativechain}

Creativechain (CREA) is a state-of-the-art project that proposes a concept for the management and protection of intellectual property by combining multiple techniques. Firstly, Decentralized Trusted Timestamping [11] is used to secure digital content by OP_RETURN transactions, which allows embedding a hash directly into the blockchain. Given a blockchain-based timestamp, it is de-facto possible to prove that work already existed at a certain point in time. Moreover, the concept provides for direct access to lawyers to punish copyright infringements. Rights of use of the works are regulated by Smart Contracts. Item data is managed on the Tarsius File System according to [34], which is a distributed hash table. Moreover, this concept makes use of decentralized identity management, which is capable of anonymity, but no further information was given how this goal is reached. The consensus protocol [35] is Proof-of-Work [9], which is not beneficial regarding the energy consumption [36].

The technical information in the whitepaper seems to be outdated as different hashing methods are used 5 CREA is based on a Bitcoin fork, and Smart Contracts are not yet implemented. Therefore only the concept of Creativechain can be considered here mainly.

\subsection{LBRY}

LBRY is an open-source protocol that is based on a distributed network of hosts and focuses on the decentralized distribution and discovery of media content addressing both media publishers and consumers. The protocol is designed to handle licensing and payment without requiring the third party. It reuses concepts from BitTorren $1^{6}$ and IPFS 7 (decentralized storage of single media bits), but adds important functionality:

Content Exploration. For both BitTorrent and IPFS,

https://bit.ly/2JDVabf

https://www.bittorrent.com/

https://ipfs.io/ 
a user is required to know a certain file's id. LBRY supports content delivery without requiring an index, similar to IPNS (name system).

Monetization/Reward. Rewarding of content publishers is an intrinsic property of the LBRY protocol. Based on their own LBC token, users automatically pay for consumed media content.

LBRY supports media upload, delivery, and consumption using both an application and their AP 8 Neither reliable usage statistics, nor a technical whitepaper does exist, but a market capitalization of 20M USD 9 indicates a strong interest.

\subsection{MILC}

Welt der Wunder $\mathrm{GmbH}$ [37], a production and broadcasting company, is expanding a blockchain-based licensing platform for high-resolution videos. The main problem that the platform wants to solve is the enormous amount of time it takes intermediaries to check and issue licenses. In cooperation with a local law firm, standardized usage rights are developed and mapped on a chain code basis (Hyperledger Fabric).

The company is currently in the crowdfunding phase (Initial Coin Offering with the Micro licensing Coin (MILC), linked to Ethereum), but already has a web-based platform in the beta stadium. Since the licensing processes for videos are very individual and complex, the platform concentrates only on the B2B market. Licenses are to be generated automatically not only for complete film files but also for individual excerpts. In principle, there is a good basis of trust and decades of experience with the granting of rights of use to film material between broadcasters and production companies. It remains to be seen whether the platform will be opened to $\mathrm{B} 2 \mathrm{C}$ market in the future.

The film material is stored locally at the respective production companies; only the digital fingerprint including metadata is held on the blockchain and references the film material. Possible misuse of licenses is not detected by the platform and is not planned for the future.

\section{7. po.et}

Po.et empowers users to record metadata and ownership information for their digital creative work. This service works by creating a digital fingerprint (proof-of-existence) with BT over the Bitcoin protocol. Additional to that Po.et includes several other metadata

\footnotetext{
${ }^{8}$ A JSON-RPC API https: / / lbryio.github.io/lbry/

${ }^{9}$ As of June 2018, https://coinmarketcap.com/ currencies/library-credit/
}

to improve discoverability for the original asset [38]. The desired outcome is to solve three main questions in case of using digital assets on the internet today: who is the owner of the digital asset? Am I allowed to use it or do I need a license? Where does this asset come from and what is its history? The project answers the first question with timestamping and metadata. For answering the second and third question, Po.et uses a badge which contains originating information about the asset, authentication and it also shows whether the free use of the content is authorized by the creator(s).

For the future, it is planned to provide various pre-existing licenses, and the possibility to create custom contracts to help the users to protect and monetize their content [38]. Another feature which the developers behind po.et have planned is the possibility to create digital marketplaces for the network participants to offer their works. For some of these actions (creating marketplaces, registering, and buying content) it is needed to use po.et tokens (POE). Potential users of this platform are publishers, editors, and content creators.

\subsection{Mediachain}

Mediachain lets users publish, explore and collaborate on different media formats and allows to build decentralized media applications. It offers a distributed media directory with different topics users can subscribe. Files and topics are distributed over the network powered by a combination of Ethereum and IPFS.

Access is possible via an API and a CLI. Although the technical documentation is thorough, Mediachain is not widely adopted yet. They present a use-case where different museums share their archives about cultural heritage using the Mediachain architecture to build public and decentralized catalog of, e.g., metadata of paintings.

\section{Summary and Discussion}

This survey serves as a snapshot into the available and planned blockchain applications in the context of licensing and distributing creative assets and intellectual property. We recognized early in the analysis that all projects following their ways and goals which is complicated for the user who is searching for protection and distribution for their digital content.

Concerning the essential functions for licensing and distribution of intellectual property, Creativechain is the only application with working features. Other systems only provide rough concepts for these use-cases. Moreover, more essentials modules, such 


\begin{tabular}{|c|c|c|c|c|c|c|c|c|}
\hline & ascribe & bernstein & binded & $\begin{array}{c}\text { Creative } \\
\text { chain }\end{array}$ & LBRY & MILC & po.et & $\begin{array}{l}\text { Media } \\
\text { chain }\end{array}$ \\
\hline Document Type & Images & Patents & Images & Multimedia & Multimedia & Video & Multimedia & Audio \\
\hline Business Type & $\mathrm{B} 2 \mathrm{C}$ & B2B & $\mathrm{B} 2 \mathrm{C}$ & $\begin{array}{c}\mathrm{C} 2 \mathrm{C} \& \\
\mathrm{~B} 2 \mathrm{~B}\end{array}$ & $\begin{array}{c}\mathrm{C} 2 \mathrm{C} \& \\
\mathrm{~B} 2 \mathrm{~B}\end{array}$ & B2B & $\mathrm{C} 2 \mathrm{C}$ & $\begin{array}{c}\text { B2B \& } \\
\text { B2C }\end{array}$ \\
\hline Reach / NOU & mid & mid & mid & high & high & low & mid & high \\
\hline Access method & $\begin{array}{c}\text { web \& } \\
\text { API }\end{array}$ & web & web & desktop & $\begin{array}{l}\text { desktop } \\
\text { \& mobile } \\
\& A P I\end{array}$ & web & $\begin{array}{c}\text { web \& } \\
\text { API }\end{array}$ & API \\
\hline License standards & own & prop. & $\begin{array}{c}\text { own \& } \\
\text { govern. }\end{array}$ & $\mathrm{CC}$ & MIT & own & own & MIT \\
\hline Audit & no & no & no & no & no & yes & no & no \\
\hline Data structure [21] & public & public & public & public & public & private & public & public \\
\hline Item storage [21] & $\begin{array}{c}\text { hash } \\
\text { on-chain }\end{array}$ & $\begin{array}{l}\text { hash } \\
\text { on-chain, } \\
\text { file DB }\end{array}$ & $\begin{array}{l}\text { hash } \\
\text { on-chain, } \\
\text { file DB }\end{array}$ & $\begin{array}{l}\text { hash } \\
\text { on-chain, } \\
\text { file } \\
\text { torrent }\end{array}$ & $\begin{array}{l}\text { hash } \\
\text { on-chain, } \\
\text { file IPFS }\end{array}$ & $\begin{array}{l}\text { hash } \\
\text { on-chain, } \\
\text { file DB }\end{array}$ & $\begin{array}{l}\text { hash } \\
\text { on-chain, } \\
\text { file } \\
\text { torrent, } \\
\text { file DB }\end{array}$ & $\begin{array}{c}\text { hash } \\
\text { on-chain, } \\
\text { file IPFS }\end{array}$ \\
\hline Consensus protocol [21] & PoW & PoW & PoW & PoW & PoW & PBFT & PoW & PoW \\
\hline Reference Impl. & Bitcoin & Bitcoin & Bitcoin & Bitcoin & Bitcoin & Hyp.Ledg. & Bitcoin & Ethereum \\
\hline Identity Management & central. & central. & central. & decentral. & decentral. & central. & central. & hybrid \\
\hline Incentives & no & no & no & yes & yes & no & no & no \\
\hline Peer-reviewed & no & no & no & no & no & no & no & no \\
\hline
\end{tabular}

Table 2. Comparing blockchain-based licensing and distribution platforms

as collaboration tools, citing and rewarding techniques, cross-solution exchanges, recommendation systems, and revision and traceability modules are either only conceptually available or not available at all. However, comprehensive copyright systems require the mentioned features, which are currently not implemented by any solution based on DLT.

The market for blockchain-based licensing and distribution applications causes confusion since there are no standards for developers to follow. Also, users can't orientate themselves with such a rule book to search for their best solution. We provide a step on the way to a standard with our list of categories which underlie for every rating of all analyzed applications (see Table 2).

Fraud and abuse of digital assets are difficult to prevent at this moment. Two examples following:

1. An individual can claim ownership with a digital fingerprint (proof-of-existence) but how others can be sure that this individual is the originator of that digital asset? If a person gets an IP of another person in some way and discloses it before the actual originator, it is not detectable who is the owner of the digital content. Due to that lack of transparency, it is always a questionable process.

2. A protected picture could be changed by just a bit to change the hash value of it; the eye often can't even see the difference between the original and the forgery. For example, a person could easily alter the hash value by changing just the RGB code of a single pixel. With that changed hash value it is far more difficult to prove fraud.

A possibility to counterattack slightly changed digital assets, as mentioned in the second example, is a perceptual hash. However, the results are not clear. Among other areas of application, perceptual hashes are used to finding cases of online copyright infringements or in digital forensics. A normal hash will change drastically when individual changes a bit of a digital file but a perceptual hash stays nearly the same, and due to that, it can recognize the similarity to a just slightly changed digital file.

The second approach for better protection of digital 
assets and standardization could be certificates for tools that means there would be a range of trusted tools from that users can choose. If you take copyright seriously, you will use trusted tools that use a certified API. If you edit and publish an image without a trusted tool, you make yourself suspicious. Kodak has taken a related approach with KodakOne [39] since the end of 2017. However, there is little information on this.

The biggest technical differences can be found in the storage methodology of the examined solutions. The hashes of the files (fingerprints) are always stored on-chain. However, the original files are stored in a central database or distributed file systems. Either in a torrent system or the IPFS. The question arises whether decentralized storage is even worthwhile if the central type is the cheapest [24]. The data must be encrypted in the IPFS, and a shared layer or even an access control mechanism must be included so that the security hole cannot be exploited. After all, as long as you know the hash of a file that is in IPFS, you will be able to download the underlying file. Sensitive files, as they are in our context, are usually not suitable for IPFS. Encryption with shared layer must be developed for this 10

Even though some of the considered solutions are privately managed, the code of the applications is completely opened to the public (Creativechain ${ }^{11}$ po.e ${ }^{12}$. This leads to more transparency and trustworthiness. Furthermore, the services may benefit from the contributions of the community.

\section{Conclusion}

In this work, we have investigated state-of-the-art blockchain-based applications that support licensing and distribution of intellectual property. We have divided the wide range of existing applications into sub-groups and focused on applications which Elsden at al. classified as belonging to the group of "property and ownership".

We selected both non-technical and technical criteria for the comparison of the applications in our review. Non-technical criteria were used to assess the range of functions that applications provide while technical criteria helped us to examine the technology used to realize the applications. Finally, we analyzed and classified eight different platforms according to the chose criteria. The resulting classification is provided as a comparison matrix.

All applications included in our review are still in

10 https://bit.1y/2GjYeUi

11 https://github.com/creativechain

12 https://github.com/poetapp an early stage of development and do not yet deliver their full functionality. Often, features are described in the white paper describing an application, but are not implemented yet. For example, in one application, the monitoring of already licensed images is unreliable, while the client software of another application exhibits problems in synchronizing the blockchain. However, all solutions provide reliable registration of digital assets and thus creating proofs-of-existence.

The domain of intellectual property protection with DLT exhibits a trend of platformization. De la Rosa et al. described this phenomenon in 2017 [2]. They named the underlying power of network effects and organizations' lack of trust in decentralized processes as major reasons for this development. Organizations often prefer to trust platforms run by people, since people can be held responsible in case of errors. However, this trend of centralization contradicts the fundamental idea of decentralized blockchains and distributed ledger technology and results in a dependency of the user on the service.

As future work, we suggest to perform a study on the feasibility of establishing a standard technology as well as a common interoperable metadata format. Having standard technologies and data formats would increase the security for users since the protected content would be detached from a particular application platform.

The summary we derive from our review is that blockchain technology is highly relevant and beneficial for automated licensing, especially of digital works, but still lacks approaches to prevent the unauthorized reuse of already licensed files.

\section{References}

[1] G. S. Lunney, S. Virginia, L. Review, N. Sep, and V. Law, "The death of copyright: Digital technology, private copying, and the digital millennium copyright act," vol. 87, no. 5, pp. 813-920, 2001.

[2] J. L. De la Rosa, V. Torres-Padrosa, A. El-Fakdi, D. Gibovic, L. Maicher, and F. Miralles, "A survey of Blockchain Technologies for Open Innovation," White Paper, no. November, pp. 1-27, 2017.

[3] C. Elsden, A. Manohar, J. Briggs, M. Harding, C. Speed, and J. Vines, "Making Sense of Blockchain Applications: A Typology for HCI," ACM SIGCHI Conference on Human Factors in Computing Systems (CHI), pp. 1-14, 2018.

[4] A. Schoenhals, T. Hepp, and B. Gipp, "Design Thinking using the Blockchain Enable Traceability of Intellectual Property in Problem-Solving Processes for Open Innovation," 2018.

[5] H. W. Chesbrough, Open innovation: The new imperative for creating and profiting from technology. Harvard Business Press, 2006.

[6] U. Lichtenthaler, "Integrated Roadmaps for Open Innovation," Cemi.Com.Au, pp. 45-50. 
[Online]. Available: http://www.cemi.com.au/sites/all/ publications/Lichtenthaler2008.pdt

[7] F. Tschorsch and B. B. Scheuermann, "Bitcoin and beyond: A technical survey on decentralized digital currencies," IEEE Communications Surveys and Tutorials, vol. 18, no. 3, pp. 2084-2123, 2016.

[8] S. Popov, "The Tangle," 2017. [Online]. Available: http://iotatoken.com/IOTA_Whitepaper.pdf

[9] S. Nakamoto, "Bitcoin: A Peer-to-Peer Electronic Cash System,"Www.Bitcoin.Org, p. 9, 2008. [Online]. Available: https://bitcoin.org/bitcoin.pdf

[10] J. Zou, B. Ye, L. Qu, Y. Wang, M. A. Orgun, and L. Li, "A Proof-of-Trust Consensus Protocol for Enhancing Accountability in Crowdsourcing Services," IEEE Transactions on Services Computing, 2018.

[11] B. Gipp, N. Meuschke, and A. Gernandt, "Decentralized Trusted Timestamping using the Crypto Currency Bitcoin,” pp. 1-6, 2015. [Online]. Available: http: //arxiv.org/abs/1502.04015

[12] M. Conoscenti, A. Vetro, and J. C. De Martin, "Blockchain for the Internet of Things: A systematic literature review," in Computer Systems and Applications (AICCSA), 2016 IEEE/ACS 13th International Conference of. IEEE, 2016, pp. 1-6.

[13] A. S. de Pedro Crespo and L. I. C. Garc $\backslash \backslash \backslash$ ia, "Stampery Blockchain Timestamping Architecture (BTA)," 2016.

[14] dock.io, "Decentralized data exchange powered by Ethereum," 2018.

[15] T. Strobl, "Landtag von Baden-Württemberg 'Potenziale und Risiken der Blockchain-Technologie'," 2017.

[16] E. E. Wood, "City of Torontos Tracey Cook thinks the future of licensing lies with blockchain," 2017. [Online]. Available: https://bit.ly/2JRDMvV

[17] E. Kane, "Is Blockchain a General Purpose Technology?" 2017.

[18] T. Swanson, "Consensus-as-a-service: a brief report on the emergence of permissioned, distributed ledger systems," Whitepaper, p. 66, 2015. [Online]. Available: http://www.ofnumbers.com/wp-content/ uploads/2015/04/Permissioned-distributed-ledgers.pdt

[19] C. Maloney, "Scam Exposed: Fraudulent Company Registers to Exploit Billion-Dollar Telegram ICO," 2018. [Online]. Available: https://bit.ly/2JVQWLt

[20] M. Swan, Melanie Swan-Blockchain_ Blueprint for a New Economy-O'Reilly Media (2015).

[21] X. Xu, I. Weber, M. Staples, L. Zhu, J. Bosch, L. Bass, C. Pautasso, and P. Rimba, "A Taxonomy of Blockchain-Based Systems for Architecture Design," Proceedings - 2017 IEEE International Conference on Software Architecture, ICSA 2017, pp. 243-252, 2017.

[22] V. Buterin, "On public and private blockchains," 82015. [Online]. Available: https://bit.ly/1UsVKV4

[23] I. Weber, X. Xu, R. Riveret, G. Governatori, A. Ponomarev, and J. Mendling, "Untrusted Business Process Monitoring and Execution Using Blockchain," in Lecture Notes in Computer Science. Springer International Publishing, 2016, pp. 329-347.

[24] X. Xu, C. Pautasso, L. Zhu, V. Gramoli, A. Ponomarev, A. B. Tran, and S. Chen, "The Blockchain as a Software Connector," 2016 13th Working IEEE/IFIP Conference on Software Architecture (WICSA), pp. 182-191, 2016.
[25] J. Weiner, “The Tangle," 2017.

[26] S. Forestier, "Blockclique: scaling blockchains through transaction sharding in a multithreaded block graph," 2018. [Online]. Available: http://arxiv.org/abs/1803. 09029

[27] I. Eyal, A. E. Gencer, E. G. G. Sirer, R. van Renesse, R. V. Renesse, and I. Nsdi, "Bitcoin-NG: A Scalable Blockchain Protocol," 2015. [Online]. Available: http://arxiv.org/abs/1510.02037

[28] J. Poon and T. Dryja, "The Bitcoin Lightning Network: Scalable Off-Chain Instant Payments," Technical Report (draft), p. 59, 2016. [Online]. Available: https: //lightning.network/lightning-network-paper.pdf

[29] BitFury Group, "Proof of Stake versus Proof of Work," vol. 2015, pp. 1-26, 2015. [Online]. Available: http://bitfury.com/content/ 5-white-papers-research/pos-vs-pow-1.0.2.pdt

[30] Z. Zheng, S. Xie, H. Dai, X. Chen, and H. Wang, "An Overview of Blockchain Technology: Architecture, Consensus, and Future Trends," in Proceedings - 2017 IEEE 6th International Congress on Big Data, BigData Congress 2017, 2017.

[31] M. Ahmed and K. Kostiainen, "Identity Aging: Efficient Blockchain Consensus," 2018.

[32] T. Mcconaghy and D. Holtzman, "Ascribe.io Whitepaper," 2015. [Online]. Available: www.ascribe.io

[33] M. Barulli, "Bernstein.io Product Deck," Tech. Rep., 2018.

[34] The Creativechain Team, "Creativechain Whitepaper," $2017 . \quad$ [Online]. Available: https://creativechain.org/wp-content/uploads/2017/ 03/Whitepaper-Creativechain-1.2.pdt

[35] L. Xu, L. Chen, Z. Gao, Y. Lu, and W. Shi, "CoC: Secure Supply Chain Management System Based on Public Ledger," 2017 26th International Conference on Computer Communication and Networks (ICCCN), pp. 1-6, 2017. [Online]. Available: http: //ieeexplore.ieee.org/document/8038514/

[36] Z. Zheng, S. Xie, H.-N. Dai, and H. Wang, "Blockchain Challenges and Opportunities : A Survey Shaoan Xie Hong-Ning Dai Huaimin Wang," International Journal of Web and Grid Services, pp. 1-24, 2016. [Online]. Available: http://inpluslab.sysu.edu.cn/files/blockchain// blockchain.pdt

[37] Welt der Wunder TV, "MILC Whitepaper," 2018. [Online]. Available: https://bit.ly/2HTc9km

[38] “po.et Whitepaper," 2018. [Online]. Available: https: //bit.ly/2ByriYz

[39] Kodak, "KodakOne White paper," 2018. [Online]. Available: https://kodakone.com/fileadmin/white_paper/ 180424_kodakone_wp.pdf

[40] European Commission, "Directive of the European Parliament and of the council on copyright in the Digital Single Market," 2016. [Online]. Available: https://eur-lex.europa.eu/legal-content/EN/ TXT/?uri=CELEX:52016PC0593 